\title{
Fabrication of Self-Aligned Graphene FETs with Low Fringing Capacitance and Series Resistance
}

\author{
Yanjie Wang, ${ }^{1}$ Bo-Chao Huang, ${ }^{1}$ Ming Zhang, ${ }^{1}$ Congqin Miao, ${ }^{2}$ \\ Ya-Hong Xie, ${ }^{2}$ and Jason C. S. Woo ${ }^{1}$ \\ ${ }^{1}$ Department of Electrical Engineering, Henry Samueli School of Engineering and Applied Science, University of California, \\ Los Angeles, Los Angeles, CA 90095-7065, USA \\ ${ }^{2}$ Department of Materials Science and Engineering, Henry Samueli School of Engineering and Applied Science, University of California, \\ Los Angeles, Los Angeles, CA 90095-7065, USA
}

Correspondence should be addressed to Bo-Chao Huang, alanhuang@ucla.edu

Received 23 July 2012; Accepted 9 August 2012

Academic Editors: L. Belostotski, J. Solsona, and E. Tlelo-Cuautle

Copyright (๑) 2012 Yanjie Wang et al. This is an open access article distributed under the Creative Commons Attribution License, which permits unrestricted use, distribution, and reproduction in any medium, provided the original work is properly cited.

Graphene FETs with top-gate and buried-gate structure has been studied. The buried-gate structure shows less fringing capacitance and more reliable contacts. High-performance graphene transistors with self-aligned buried gates have been fabricated. The graphene transistor shows field-effect mobility of over $6,000 \mathrm{~cm}^{2} / \mathrm{V} \cdot \mathrm{s}$ according to the transconductance measurement. The contact resistance and intrinsic mobility have been extracted from both curve fitting and transfer length measurement, and the two results agree well. This result paves the way of high-quality graphene transistor technology for the RF application.

\section{Introduction}

As a two-dimensional material composed of a layer of carbon atoms packed in a honeycomb crystal lattice, graphene has attracted much attention in recent years [1-4]. Graphene shows great potential as a candidate for highfrequency devices application due to its high intrinsic carrier mobility $\left(>10,000 \mathrm{~cm}^{2} / \mathrm{V} \cdot \mathrm{s}\right)[5]$, large saturation velocity $\left(5.5 \times 10^{7} \mathrm{~cm} / \mathrm{s}\right)[6]$, and high on-state current density $(>3 \mathrm{~A} / \mathrm{mm})[7,8]$. Graphene-based field-effect transistors (FETs) with a cut-off frequency of $300 \mathrm{GHz}[8,9]$ have been achieved. High-mobility grapheme FET $\left(6,000 \mathrm{~cm}^{2} / \mathrm{V} \cdot \mathrm{s}\right.$ and $23,600 \mathrm{~cm}^{2} / \mathrm{V} \cdot \mathrm{s}$ before and after the extraction of the contact resistance) and different synthesis methods of graphene (epitaxial graphene on $\mathrm{SiC}, \mathrm{CVD}$ grapheme, and mechanical exfoliated graphene) have been reported $[7,10,11]$. Scalable synthesis of graphene on patterned $\mathrm{Ni}$ has been reported [12].

Parasitic capacitance, series resistance, and mobility are the major factors to influence the high-frequency performance of graphene FETs. Self-aligned technique will minimize the series resistance and overlap capacitance, as well as simplify the lithography process. Top-gate graphene FETs with self-aligned structure have been reported by many groups $[8,13,14]$. In these structures, the gate dielectric profile will separate the thin source/drain metal during the source/drain evaporation. However, since the gate and source/drain are on the same side of graphene, this will address several issues for the devices. First, the fringing capacitance in these structures could become an important portion for short-channel devices. Secondly, these structures will restrict the thickness of source/drain otherwise, the source/drain will become shorted. The thin source/drain contact will increase the series resistance and also raise a reliability issue for the RF application of graphene FET since the thin source/drain contact is more vulnerable under large current density.

Graphene FET with buried-gate structure has been published [15-17]. Compared with the top-gate structure, the buried-gate structure enables gate stack and source/drain on different sides, which can reduce the negative effect from the top-gate structures. First, the buried-gate structure can reduce the fringing capacitance between gate and source/ drain. Second, thicker source/drain metal could be used 


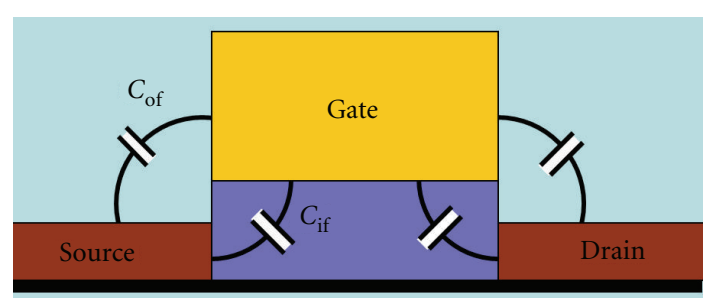

(a)

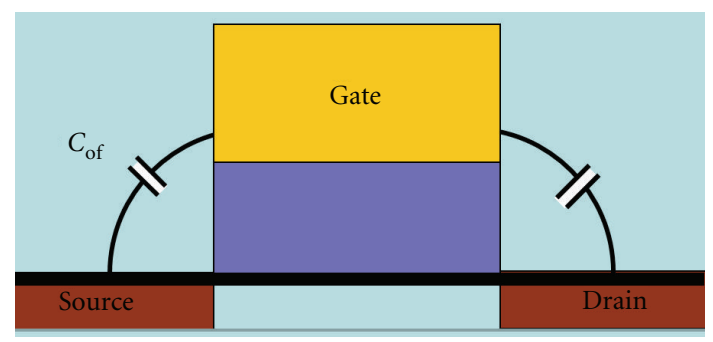

(b)

FIGURE 1: Fringing capacitance in for top-gated (a) and buriedgated (b) structure.

to improve the reliability and reduce the series resistance. Buried-gate graphene FET with "T-" gate structure has been fabricated [15], which will reduce the gate resistance and therefore improve the unit power gain frequency. In light of the benefits of buried-gated structure, buried-gated graphene FETs with self-aligned structure is studied in this paper.

\section{Comparison between Buried- and Top-Gate Structure}

The unity-current-gain frequency $f_{T}$ and unity-power-gain frequency $f_{\max }$ are two important figures of merits for RF transistors. The parasitic resistances and capacitance will degrade $f_{T}$ and $f_{\max }$ in FET. In graphene FET, the parasitic capacitance composed mainly of direct overlap capacitance and fringing capacitance (between gate and source/drain). Self-aligned process can minimize the direct overlap capacitance and parasitic resistance. In self-aligned structures, the fringing capacitance between gate and source/drain becomes the major parasitic capacitance in the devices. This fringing capacitance composed of outer fringing capacitance $C_{\mathrm{of}}$ and inner fringing capacitance $C_{\mathrm{if}}$, as shown in Figure 1 . For top-gated devices (Figure 1(a)), both $C_{\text {of }}$ and $C_{\text {if }}$ exist. For buried-gated devices (Figure 1(b)), the inner fringing capacitance become negligible since the carriers in graphene will effectively shield any electrostatic coupling between the gate and the inner edges of the source or drain junction [18]. Therefore, the influence of the source/drain metal thickness on the fringing capacitance can be ignored for devices' structure of Figure 1(b).

The fringing capacitance for top-gate and buried-gate devices has been simulated with finite element method. In this simulation, the parameters used here are from [13], where the gate metal thickness is $60 \mathrm{~nm}$, gate dielectric

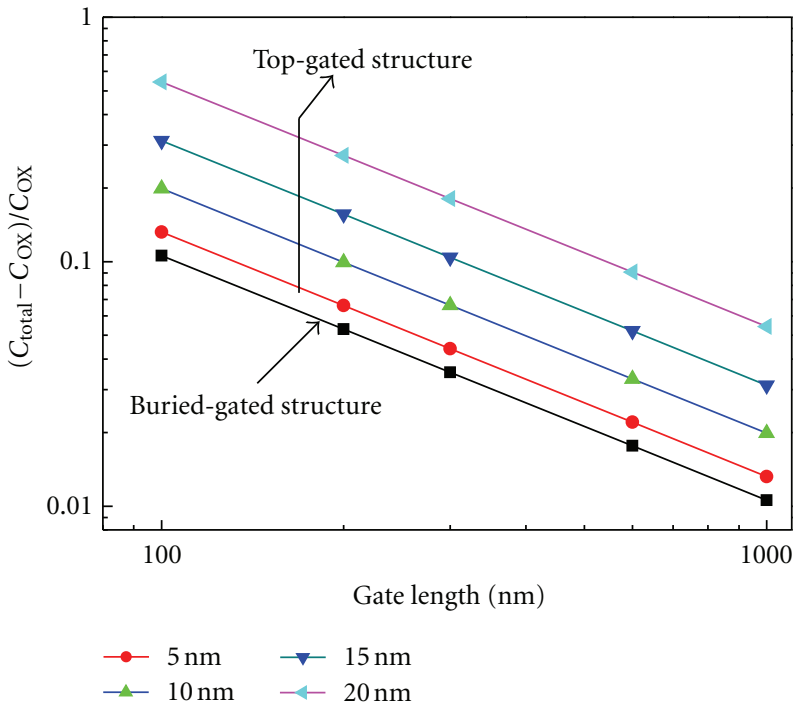

FIgure 2: The fringing capacitance at different gate length and source drain thickness for buried-and top-gated devices.

is $25 \mathrm{~nm} \mathrm{Al} \mathrm{Al}_{2} \mathrm{O}_{3}$, and the source/drain metal thickness is $10 \mathrm{~nm}$. $C_{\text {total }}$ is the total gate capacitance and $C_{\mathrm{OX}}$ is the gate oxide capacitance calculated from parallel plate capacitance relation.

Figure 2 plotted the fringing capacitance at different gate length for buried-and top-gated devices. It can be seen that the fringing capacitance becomes an unnegligible capacitance source especially for short channel devices. For $100 \mathrm{~nm}$ channel length and $10 \mathrm{~nm}$ thick source/drain contact metal, the fringing capacitance is about $20 \%$ and $10.6 \%$ of the gate capacitance for top-gated and buried-gate structure, correspondingly. In this case, the buried-gate structure reduced the fringing capacitance by $47 \%$ compared with the top-gate structure. The influence of the source/drain thickness on the fringing capacitance is also plotted in Figure 2. For buriedgate devices, the fringing capacitance equals that of top-gate devices at zero source/drain thickness and will not increase with increasing of the source/drain thickness. For top-gate devices with $100 \mathrm{~nm}$ channel length, the fringing capacitance ratio increases from $13.2 \%$ to $54 \%$ with the thickness of source drain increase from $5 \mathrm{~nm}$ to $20 \mathrm{~nm}$.

In order to avoid current shortage through sidewall region, thin source/drain is used for the self-aligned topgate device. Current published results for these devices all have the thickness of the source/drain of around $10 \mathrm{~nm}$ $[13,14]$. We have studied the dependence of the electrode breakdown with the metal thickness, which is shown in Figure 3. Figure 3(a) shows the breakdown current for $10 \mathrm{~nm}$ (Ti $0.5 \mathrm{~nm} / \mathrm{Pd} 9.5 \mathrm{~nm}$ ) and $30 \mathrm{~nm}(\mathrm{Ti} 0.5 \mathrm{~nm} / \mathrm{Pd} 20 \mathrm{~nm} / \mathrm{Au}$ $9.5 \mathrm{~nm}$ ) metal. The break-down current for $10 \mathrm{~nm}$ metal is about $3.17 \mathrm{~mA} / \mu \mathrm{m}$, and the breakdown current for $30 \mathrm{~nm}$ metal is about $8.2 \mathrm{~mA} / \mu \mathrm{m}$. Since it has been reported that the on-state current of graphene FET can reach 1.5 to $3 \mathrm{~mA} / \mu \mathrm{m}$ $[7,8]$, the break-down current for $10 \mathrm{~nm}$ metal is close to the on-state current of graphene FET. This will raise the reliability issue of the graphene FET which limits the lifetime 


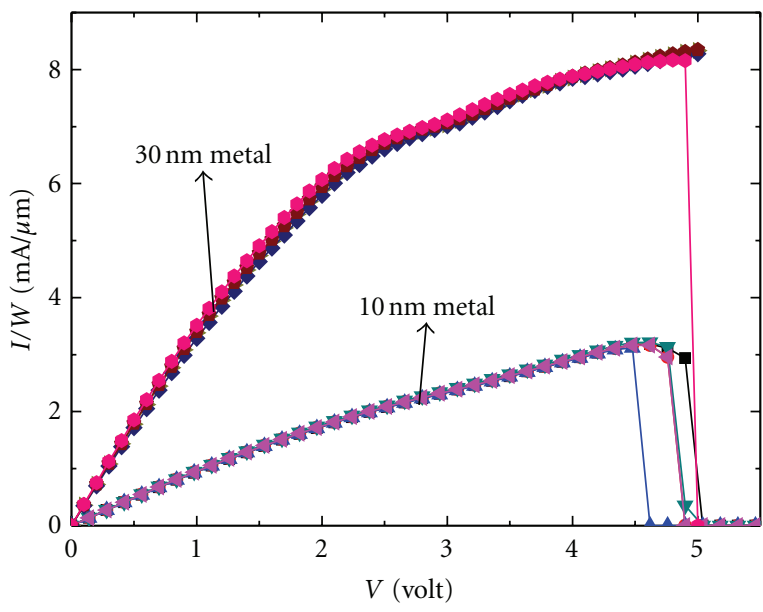

(a)

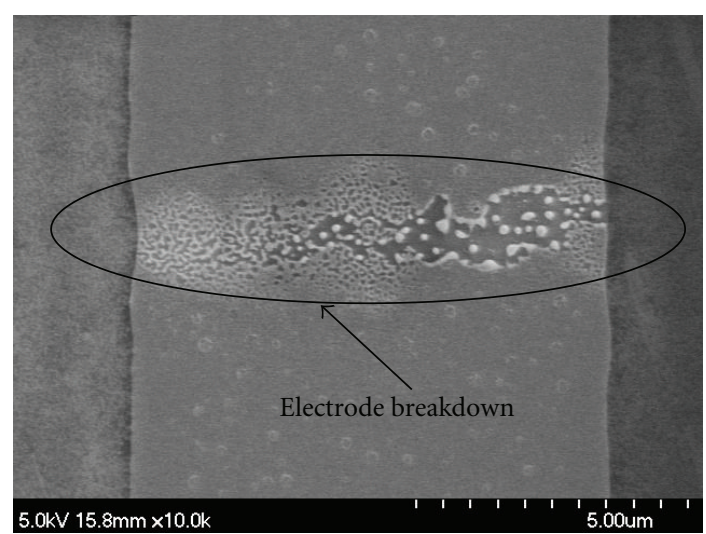

(b)

FIgURE 3: Electrode break-down current for $10 \mathrm{~nm}$ and $30 \mathrm{~nm}$ metal.

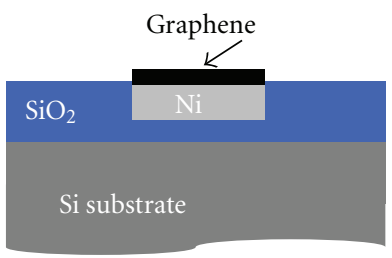

Channel definition

(a)

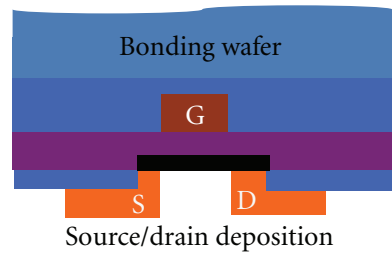

(f)

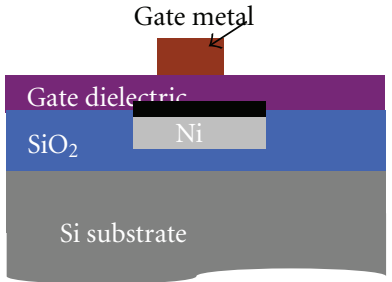

Gate stack formation

(b)

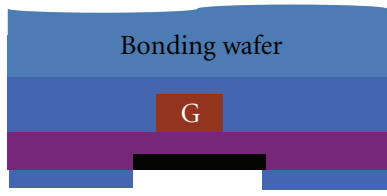

$\mathrm{SiO}_{2}$ and $\mathrm{Ni}$ etching

(e)

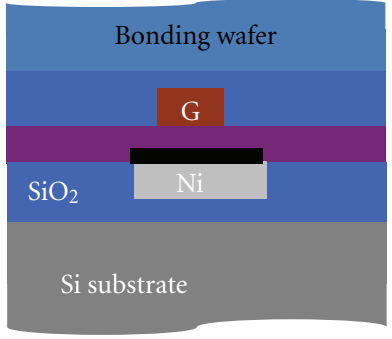

Wafer bonding

(c)

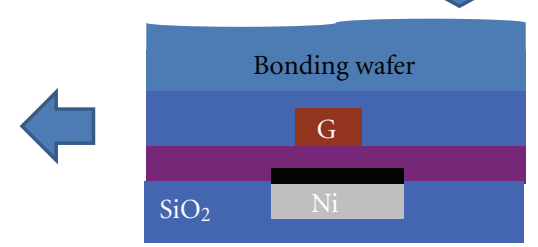

Si substrate etching

(d)

FIgURE 4: Process flow of self-aligned graphene FET.

of these devices. Figure 3(b) is an SEM picture which shows the electrode breakdown of $10 \mathrm{~nm}$ metal (Ti $0.5 \mathrm{~nm} / \mathrm{Pd}$ $9.5 \mathrm{~nm}$ ). There is a clear region that the electrode burned and became noncontinuous, which make the electrode to be opened. Also bubbles appear on the electrode outside the break-down region which indicates the electrode outside of the breakdown region is also damaged.

We have demonstrated a self-aligned process to fabricate graphene FET in order to reduce the fringing capacitance and source drain resistance as well as improve the reliability of the contact.

\section{Materials and Device Fabrication}

The process of fabricating self-aligned buried-gate graphene FET is discussed below. The channel graphene material was synthesized from patterned Ni substrate by chemical vapor deposition (CVD) method with methane catalyst [12]. Gra-phene film was first patterned by oxygen plasma etching to define the channel width (Figure 4(a)). Then, $15 \mathrm{~nm} \mathrm{SiO} 2$ (with e-beam evaporation) and $10 \mathrm{~nm} \mathrm{Al}_{2} \mathrm{O}_{3}$ (with atomic layer deposition) were deposited on top of graphene to form gate dielectric. The equivalent oxide thickness (EOT) of the gate dielectric was found to be $20 \mathrm{~nm}$, according to capacitance-voltage measurement. The metal gate was processed with e-beam evaporation of $\mathrm{Ti} / \mathrm{Au} / \mathrm{Ti}(5 / 50 / 5 \mathrm{~nm})$ and etching (Figure 4(b)). After this step, graphene and the gate stack were transferred with wafer bonding and etch-back method [12] (Figures 4(c), 4(d), and 4(e)). A back-side (glass wafer side) exposure was performed with the buried-gate electrode as the photomask to make the source and drain region self-aligned with gate electrode. The source/drain 


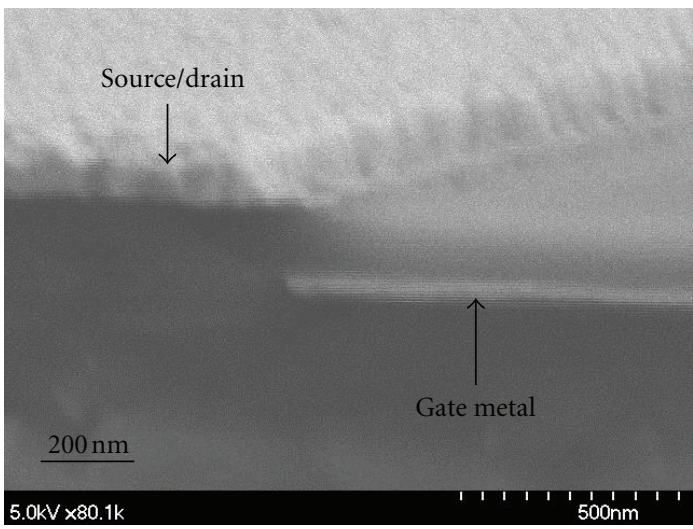

(a)

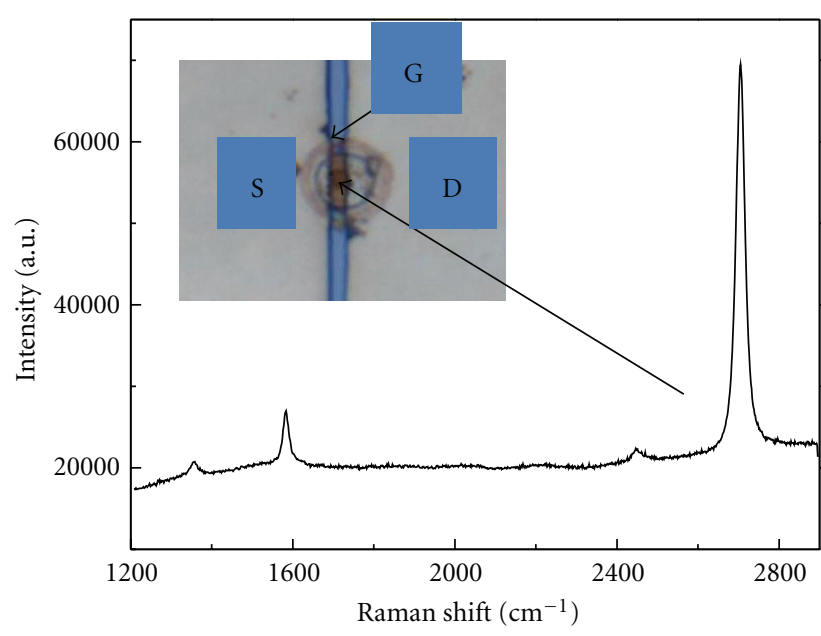

(b)

FIGURE 5: The cross-section of self-aligned structure and the Raman spectra on graphene channel.

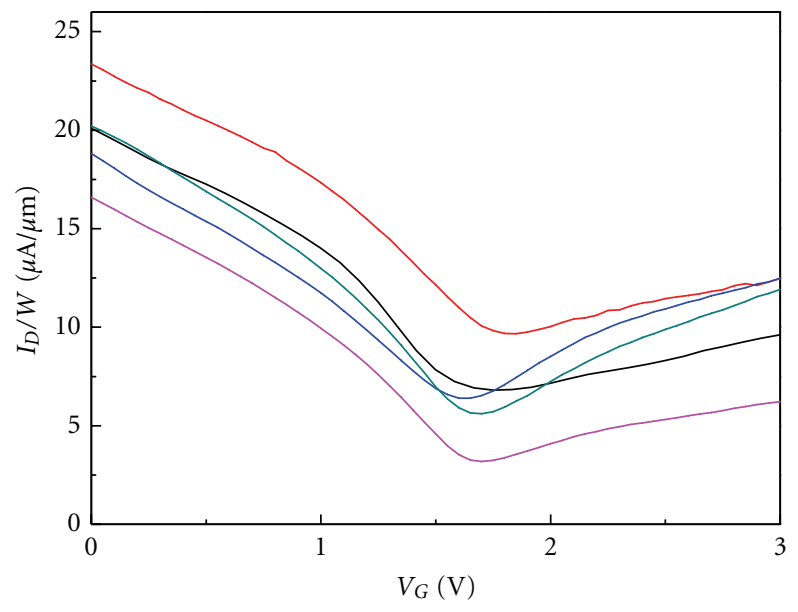

FIGURE 6: Measured transfer curves of $1.5 \mu \mathrm{m}$ gate-length graphene FETs at $V_{D}=0.01 \mathrm{~V}$.

metal pads $(0.5 \mathrm{~nm} \mathrm{Ti} / 60 \mathrm{~nm} \mathrm{Pd} / 60 \mathrm{~nm} \mathrm{Au})$ were then formed by e-beam evaporation and lift-off process (Figure $4(\mathrm{f})$ ). Here we chose $\mathrm{Ti} / \mathrm{Pd} / \mathrm{Au}$ as the contact metal because of its low contact resistivity with graphene $[19,20]$. Device fabrication is completed through open-the-gate contact to expose buried-gate metal pads.

\section{Results and Discussion}

The cross-section scanning electron microscope (SEM) picture of a graphene FET showing self-aligned source/drain with the gate metal is shown in Figure 5(a). The Raman spectra on the channel region of graphene FETs is shown in Figure 5(b), with an inset of optical microscope (OM) image. The intensity of the $D$-peak $\left(1354 \mathrm{~cm}^{-1}\right)$ is negligible compared to that of $G$-peak $\left(1581 \mathrm{~cm}^{-1}\right)$, indicating that disorder in these samples is negligible. The $2 D$ peak at $\sim 2705 \mathrm{~cm}^{-1}$ shows single Lorentzian distribution with full width at half maximum (FWHM) of $30 \mathrm{~cm}^{-1}$, demonstrating the absence of interlayer coupling. The peak intensity ratio of $I_{2 D} / I_{G}$ is about 7 . This characteristic indicates that singlelayer graphene was transferred onto gate dielectric with little material damage.

Electrical measurement was performed at room temperature. Figure 6 shows five transfer curves of graphene FETs with gate length equals gate width equals $1.5 \mu \mathrm{m}$, which were measured at $V_{D}=0.01 \mathrm{~V}$. The gate leakage currents were on the order of $<40 \mathrm{pA} / \mu \mathrm{m}$, which is negligible in the device characterization presented here. Generally, the devices shows p-channel operation at $V_{G}=0 \mathrm{~V}$, and the ambipolar behavior was observed with the Dirac point located between 1.6 to $1.8 \mathrm{~V}$ of gate voltage. The hole branch current is typically larger than electron branch current, which translates into field effect mobility of 5000 to $6000 \mathrm{~cm}^{2} / \mathrm{V} \cdot \mathrm{s}$ derived from transconductance method $\left(\mu_{\mathrm{DEV}}=g_{m} L / V_{D} W C\right)$.

Figure $7(\mathrm{a})$ shows measured $I_{D}-V_{G}$ characteristics of a graphene FET at three top-gate voltages: $-1 \mathrm{~V}, 0.15 \mathrm{~V}$, and $1.3 \mathrm{~V}$. The gate length $\left(L_{G}\right)$ and the channel width $(W)$ are both $1.5 \mu \mathrm{m}$. The gate bias is below $1.3 \mathrm{~V}$, displaying pFET hole-like conduction. Under different gate bias, the film conductance varies from 1150 to $3900 \mu \mathrm{S}$ per square calculated at $0.1 \mathrm{~V}$ drain voltage (without taking contact resistance into account). The output characteristics indicate that this device can deliver a large on-current of $1.5 \mathrm{~mA} / \mu \mathrm{m}$ at a drain voltage of $1 \mathrm{~V}$.

The dotted lines in Figure 7(b) shows transfer characteristics of the graphene FET. The gate leakage current was in the range of approximately $30 \mathrm{pA}$ per micrometer or less. The Dirac point was reached at $V_{G}=1.7 \mathrm{~V}$. Under $0.01 \mathrm{~V}$ drain voltage, the on-state current at $V_{G}=0 \mathrm{~V}$ was $20.2 \mu \mathrm{A} / \mu \mathrm{m}$. The off-state current was $5.6 \mu \mathrm{A} / \mu \mathrm{m}$ at $V_{G}=1.7 \mathrm{~V}$, yielding an $I_{\text {on }} / I_{\text {off }}$ ratio of 3.6. The maximum transconductance $g_{m}$ is $21 \mu \mathrm{S} / \mu \mathrm{m}$ for holes and $10 \mu \mathrm{S} / \mu \mathrm{m}$ for electrons; this translates into field effect mobility of $6,100 \mathrm{~cm}^{2} / \mathrm{V} \cdot \mathrm{s}$ for holes and $3,000 \mathrm{~cm}^{2} / \mathrm{V} \cdot \mathrm{s}$ for electrons $\left(\mu_{\mathrm{DEV}}=g_{m} L / V_{D} W C\right)$. 


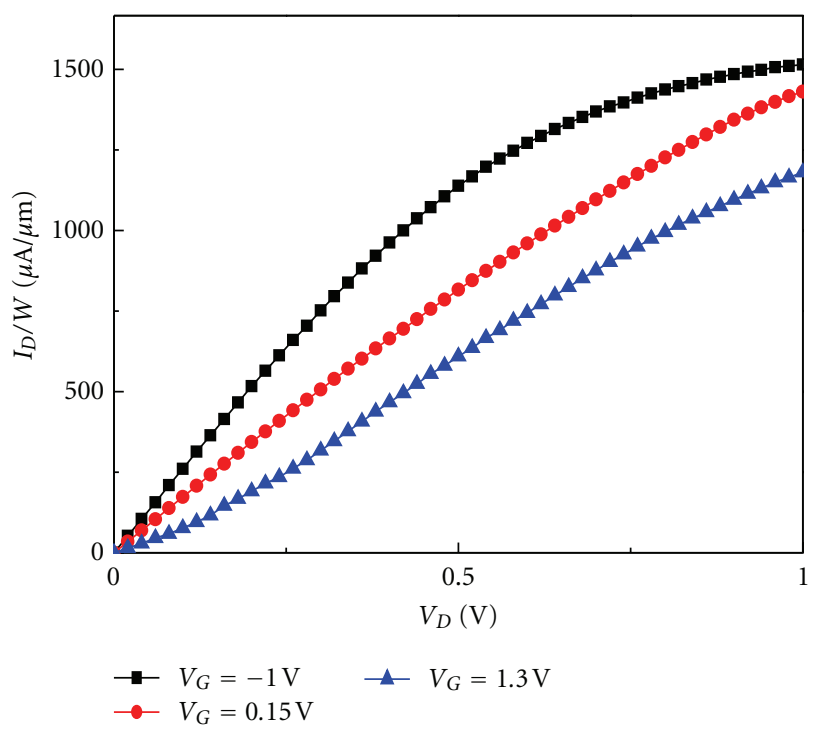

(a)

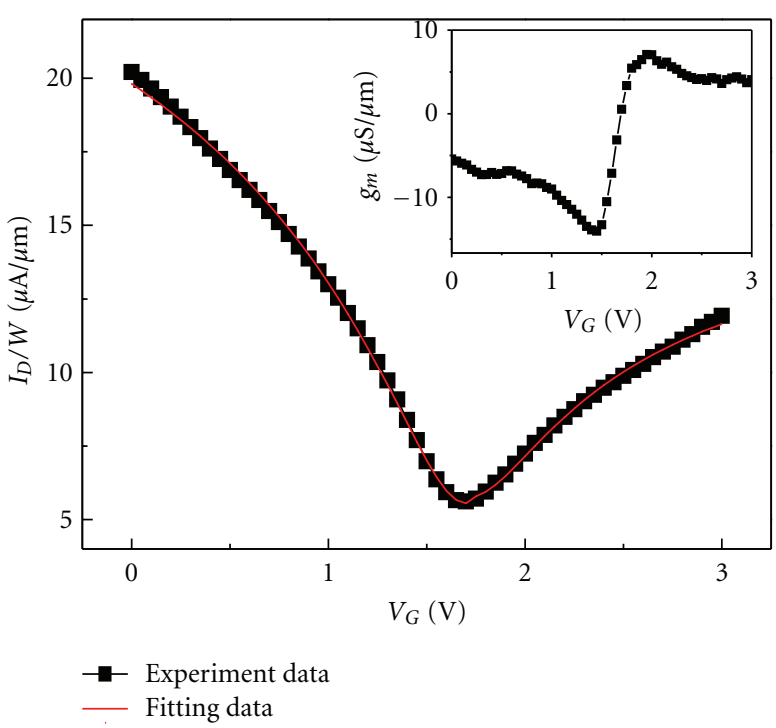

(b)

Figure 7: Output $I_{D}-V_{D}$ (a) and transfer $I_{D}-V_{G}$ characteristics (b) of graphene FET.

The electron-hole asymmetry may partially result from the series resistance of the source drain contact [21]. Pd may dope graphene to be $\mathrm{p}$-type which will favor hole conduction and restrict the current injection of electron branch, which results in a low electron mobility extracted from $g_{m}$ method.

There are many factors which will cause underestimate of the mobility extracted from the transconductance, such as parasitic resistance in source/drain region and the existence of traps in the channel region [22]. In order to get a more accurate mobility value, we subtracted the contact resistance by fitting the measured data with the following relation [13, 23]:

$$
R_{\text {Total }}=2 R_{\mathrm{C}}+\frac{L}{W e \mu \sqrt{n_{0}^{2}+n^{2}}},
$$

where $R_{\text {Total }}=V_{D} / I_{D}$ is the total resistance of the device, $e$ is the electron charge, $L$ is channel length, $W$ is channel width, $n$ is the carrier concentration induced by top-gated bias, $n_{0}$ is the residual carrier concentration of graphene, $\mu$ is the intrinsic field-effect mobility of top-gated graphene channel, and $R_{\mathrm{C}}$ is the metal/graphene contact resistance. In order to take the electron-hole asymmetry into consideration, we assigned different contact resistance for electron and hole branch on the transfer characteristics.

The fitted results (Figure 7(b)) give a mobility of $\mu_{h}=$ $25,735 \mathrm{~cm}^{2} / \mathrm{V} \cdot \mathrm{s}$ for holes and $\mu_{e}=26,538 \mathrm{~cm}^{2} / \mathrm{V} \cdot \mathrm{s}$ for electrons. The contact resistance $\left(R_{\mathrm{C}} \cdot W\right)$ was found to be $306 \Omega$. $\mu \mathrm{m}$ for electron and $153 \Omega \cdot \mu \mathrm{m}$ for hole. According to the fitting results, the mobility for electrons and holes is similar while the contact resistance for holes is about twice of that for electrons. This indicates that the asymmetry properties for electron-hole mainly originated from the contact resistance difference between electron and hole, rather than that of mobility.

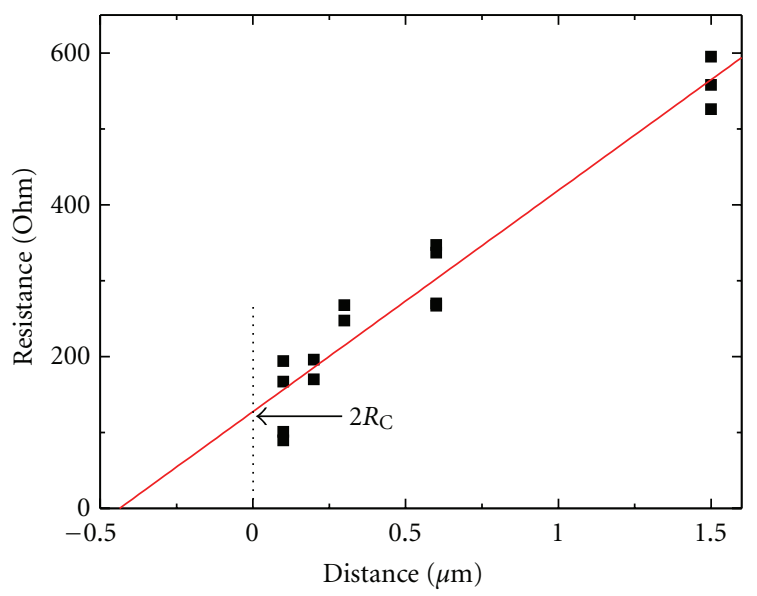

FIGURE 8: Plot of the total resistance of transistors under different channel length.

To further proof the validity of the curve fitting, we performed transfer length measurement (TLM) on graphene FET with different contact space $(100 \mathrm{~nm}, 200 \mathrm{~nm}, 300 \mathrm{~nm}$, $600 \mathrm{~nm}$, and $1500 \mathrm{~nm}$ ). A plot of the total resistance versus the contact space is shown in Figure 8. Here the graphene width is $1.5 \mu \mathrm{m}$. A linear fitting was performed to extrapolate the contact resistance, which give the total contact resistance of $2 R_{\mathrm{C}}=127 \Omega$, with the slope giving the sheet resistance of graphene of $R_{\mathrm{SH}}=436 \Omega$. Since the graphene resistance underneath the metal contact could be different with the sheet resistance of graphene between the contacts, the TLM method cannot extrapolate the transfer length and specific contact resistivity. The normalized contact resistance $R_{\mathrm{C}} W$ is $95 \Omega \mu \mathrm{m}$, which is $38 \%$ smaller than the value extracted 
from the curve fitting $(153 \Omega \cdot \mu \mathrm{m})$. The possible reasons which result in this difference could come from the following factors. First, the TLM method gives a statistical result on many data points, which could be different from a single transistor shown in Figure 7. Second, The TLM measurement was performed at zero gate voltage and the curve fitting in Figure 7 was performed with gate voltage from 0 to $1.7 \mathrm{~V}$. At 0 gate voltage there are more holes in the channel than the other positive gate voltages, which will give a smaller contact resistance. Taking these factors into consideration, we claim that the curve fitting method gives a reasonable extraction of the contact resistance, as well as the mobility.

\section{Conclusion}

We have fabricated high-performance graphene FETs with self-aligned buried-gates. The buried-gate structure offers following benefits compared with the top-gate structures: the buried-gate structures have lower fringing capacitance, which is due to larger vertical spaces between the source/ drain contact and the gate metal as well as the suppressed inner fringing capacitance by the channel carrier. On the other hand, the source/drain contact metal is more robust under large current operation since thicker source/drain could be used. The maximum field-effect mobility determined by transconductance method was found to be $6,100 \mathrm{~cm}^{2} / \mathrm{V} \cdot \mathrm{s}$ and over $25,000 \mathrm{~cm}^{2} / \mathrm{V} \cdot \mathrm{s}$ at room temperature before and after subtraction of contact resistance. The asymmetry properties in the $I_{D}-V_{G}$ curve for electrons and holes branches were found to be primarily due to the contact resistance difference. The contact resistance of electrode/ graphene was also measured from TLM method, which gives a reasonable agreement with the curve fitting result. This result provides a possible way to scalable fabricate highperformance graphene transistors for RF applications from CVD graphene.

\section{Acknowledgments}

This work was supported in part by the Defense Advanced Research Projects Agency under the Space and Naval Warfare Systems Center San Diego under Contract N66001-08-C2047 (CERA). The device fabrication was performed at the Nanoelectronics Research Facility, University of California, Los Angeles.

\section{References}

[1] M. I. Katsnelson, "Graphene: carbon in two dimensions," Materials Today, vol. 10, no. 1-2, pp. 20-27, 2007.

[2] K. S. Novoselov, A. K. Geim, S. V. Morozov et al., "Two-dimensional gas of massless Dirac fermions in graphene," Nature, vol. 438, no. 7065, pp. 197-200, 2005.

[3] P. Avouris, "Graphene: electronic and photonic properties and devices," Nano Letters, vol. 10, no. 11, pp. 4285-4294, 2010.

[4] G. H. P. Rafael, Vargas-Bernal, VLSI Design, Esteban TleloCuautle and Sheldon X.D. an(Ed), 2012.
[5] K. I. Bolotin, K. J. Sikes, Z. Jiang et al., "Ultrahigh electron mobility in suspended graphene," Solid State Communications, vol. 146, no. 9-10, pp. 351-355, 2008.

[6] I. Meric, M. Y. Han, A. F. Young, B. Ozyilmaz, P. Kim, and K. L. Shepard, "Current saturation in zero-bandgap, top-gated graphene field-effect transistors," Nature Nanotechnology, vol. 3, no. 11, pp. 654-659, 2008.

[7] J. S. Moon, D. Curtis, M. Hu et al., "Epitaxial-graphene RF field-effect transistors on Si-face $6 \mathrm{H}-\mathrm{SiC}$ substrates," IEEE Electron Device Letters, vol. 30, no. 6, pp. 650-652, 2009.

[8] L. Liao, Y. C. Lin, M. Bao et al., "High-speed graphene transistors with a self-aligned nanowire gate," Nature, vol. 467, no. 7313, pp. 305-308, 2010.

[9] Y. Q. Wu, D. B. Farmer, A. Valdes-Garcia et al., "Record high RF performance for epitaxial graphene transistors," in Proceedings of the IEEE International Electron Devices Meeting (IEDM '11), pp. 23.8.1-23.8.3, 2011.

[10] X. Li, W. Cai, J. An et al., "Large-area synthesis of high-quality and uniform graphene films on copper foils," Science, vol. 324, no. 5932, pp. 1312-1314, 2009.

[11] L. Liao, J. Bai, Y. Qu et al., "High- $\kappa$ oxide nanoribbons as gate dielectrics for high mobility top-gated graphene transistors," Proceedings of the National Academy of Sciences of the United States of America, vol. 107, no. 15, pp. 6711-6715, 2010.

[12] Y. Wang, C. Miao, B. C. Huang et al., "Scalable synthesis of graphene on patterned Ni and transfer," IEEE Transactions on Electron Devices, vol. 57, no. 12, pp. 3472-3476, 2010.

[13] D. B. Farmer, Y. M. Lin, and P. Avouris, "Graphene field-effect transistors with self-aligned gates," Applied Physics Letters, vol. 97, no. 1, Article ID 013103, 2010.

[14] J. Bai, L. Liao, H. Zhou et al., "Top-gated chemical vapor deposition grown graphene transistors with current saturation," Nano Letters, vol. 11, no. 6, pp. 2555-2559, 2011.

[15] H. Shu-Jen, A. Valdes-Garcia, A. A. Bol et al., "Graphene technology with inverted-T gate and RF passives on $200 \mathrm{~mm}$ platform," in Proceedings of the IEEE International Electron Devices Meeting (IEDM '11), pp. 2. 2. 1-2. 2. 4, 2011.

[16] I. Meric, C. R. Dean, H. Shu-Jen et al., "High-frequency performance of graphene field effect transistors with saturating IV-characteristics," in Proceedings of the IEEE International Electron Devices Meeting (IEDM '11), pp. 2. 1. 1-2. 1. 4, 2011.

[17] W. Yanjie, H. Bo-chao, Z. Ming, C. Miao, Y. H. Xie, and J. C. S. Woo, "High performance graphene FETs with selfaligned buried gates fabricated on scalable patterned nicatalyzed graphene," in Proceedings of the Symposium on VLSI Technology (VLSIT'11), pp. 116-117, 2011.

[18] T. Mueller, F. Xia, M. Freitag, J. Tsang, and P. Avouris, "Role of contacts in graphene transistors: a scanning photocurrent study," Physical Review B, vol. 79, no. 24, Article ID 245430, 2009.

[19] B. C. Huang, M. Zhang, Y. Wang, and J. Woo, "Contact resistance in top-gated graphene field-effect transistors," Applied Physics Letters, vol. 99, no. 3, Article ID 032107, 2011.

[20] Y. Wang, B.-C. Huang, M. Zhang, C. Miao, Y.-H. Xie, and J. C. S. Woo, "High performance graphene FETs with selfaligned buried gates fabricated on scalable patterned nicatalyzed graphene," in Proceedings of the Symposium on VLSI Technology (VLSIT'11), pp. 116-117, 2011.

[21] B. Huard, N. Stander, J. A. Sulpizio, and D. GoldhaberGordon, "Evidence of the role of contacts on the observed electron-hole asymmetry in graphene," Physical Review B, vol. 78, no. 12, Article ID 121402, 2008. 
[22] J. Zhu, R. Jhaveri, and J. C. S. Woo, "The effect of traps on the performance of graphene field-effect transistors," Applied Physics Letters, vol. 96, no. 19, Article ID 193503, 2010.

[23] S. Kim, J. Nah, I. Jo et al., "Realization of a high mobility dualgated graphene field-effect transistor with $\mathrm{Al} 2 \mathrm{O} 3$ dielectric," Applied Physics Letters, vol. 94, no. 6, Article ID 062107, 2009. 

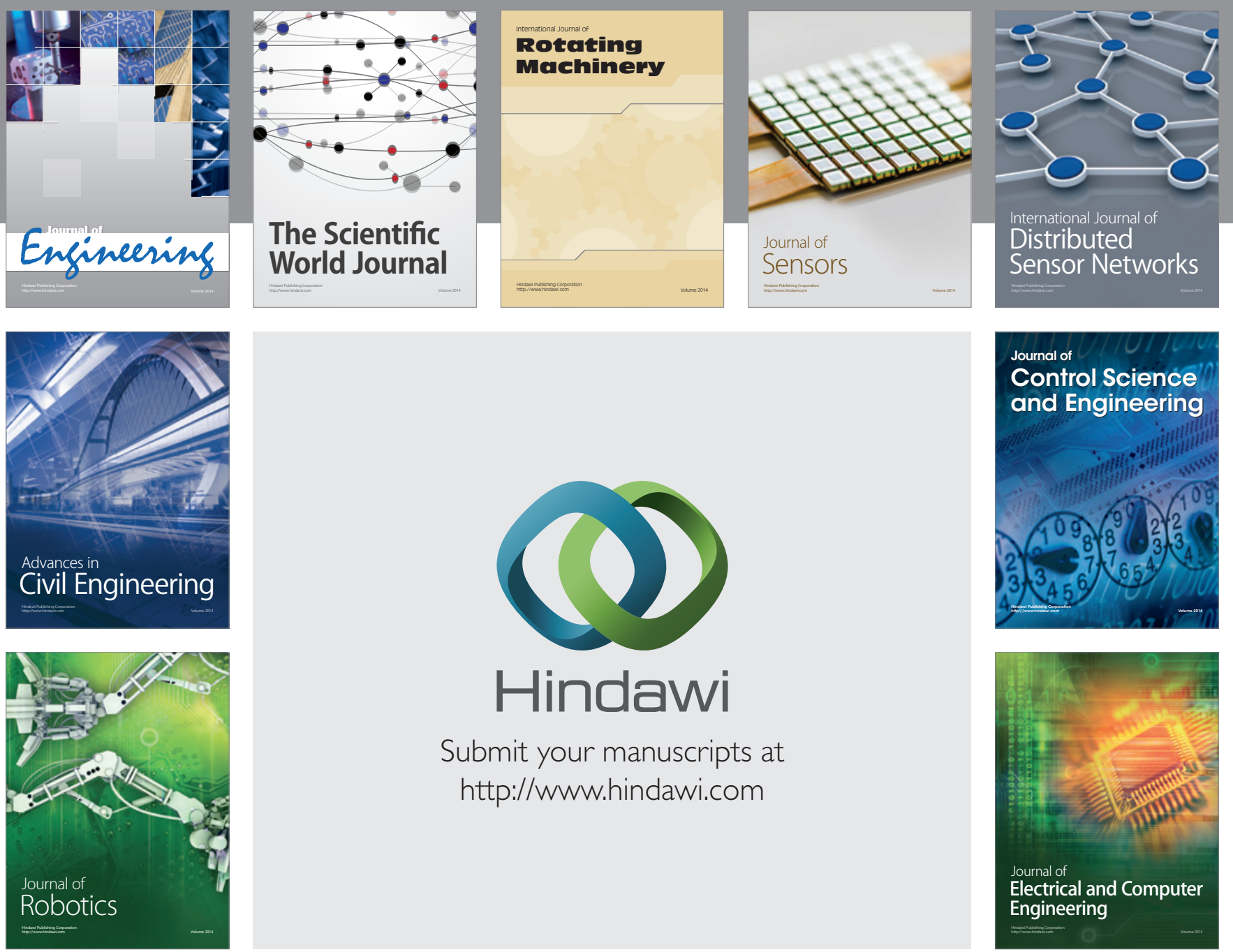

Submit your manuscripts at

http://www.hindawi.com
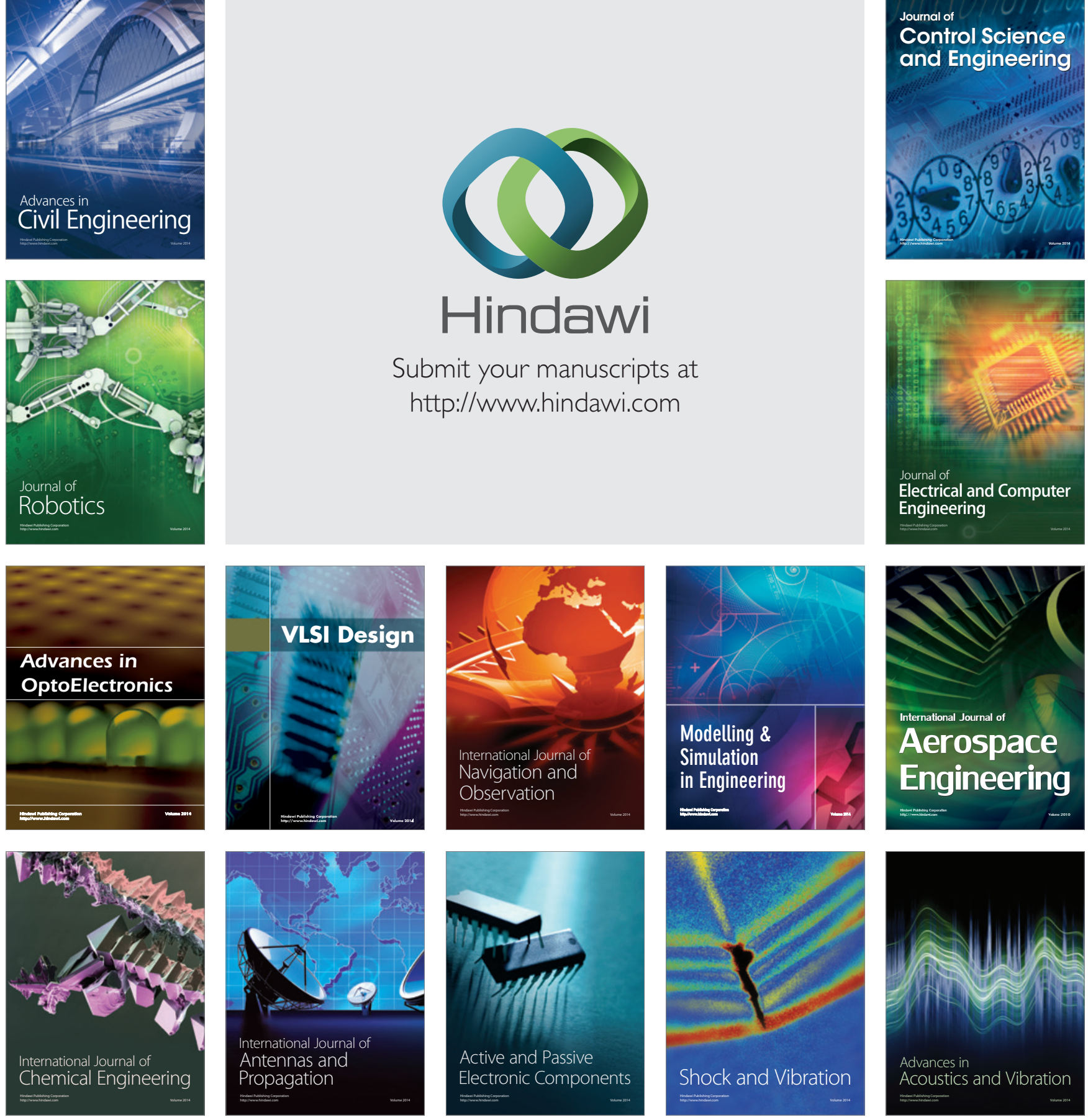